\title{
Amniotic fluid brain-specific proteins are biomarkers for spinal cord injury in experimental myelomeningocele
}
A. Petzold*
D. Stiefel ${ }^{\dagger}$
A. J. Copp

December 13, 2009

*Department of Neuroimmunology, Institute of Neurology, Queen Square, London, WC1N 3BG, United Kingdom. Fax: +44 207837 8553, E-mail: a.petzold@ion.ucl.ac.uk

†Department of Surgery, University Children's Hospital Zürich, Steinwiesstr. 75, 8032 Zürich, Switzerland \& Neural Development Unit, Institute of Child Health, University College London, 30 Guilford Street, London, United Kingdom

$\ddagger$ Neural Development Unit, Institute of Child Health, University College London, 30 Guilford Street, London, United Kingdom 


\begin{abstract}
Myelomeningocele (MMC), the most severe form of spina bifida (SB), causes neurological deficit. Injury to the spinal cord is thought to begin in utero. We investigated whether brain-specific proteins (BSP) would enable us to monitor the development of MMC-related tissue damage during pregnancy in an animal model with naturallyoccurring SB (curly tail/loop tail mouse, $\mathrm{n}=256$ ). Amniotic fluid levels of the neurofilament heavy chain (NfH), GFAP and S100B were measured by standard ELISA techniques. The amniotic fluid levels of all BSP were similar in SB and control mice on embryonic days (E) 12.5 and 14.5, whereas a significant increase was observed for GFAP in SB mice on E16.5. All BSP were significantly elevated in SB mice on E18.5. The rapid increase of GFAP which is parallelled by a moderate increase in $\mathrm{NfH}$ and $\mathrm{S100B}$ suggests that spinal cord damage starts to accelerate around E16.5. The macroscopic size of the $\mathrm{MMC}$ was related to $\mathrm{NfH}$ level on E16.5 and E18.5, suggesting that axonal degeneration is most severe in large MMC. Amniotic fluid BSP measurements may provide important information for balancing the risks and benefits to mother and child of in utero surgery for myelomeningocele.
\end{abstract}

Keywords neurofilaments, $\mathrm{NfH}$, glial fibrillary acidic protein, GFAP, S100B, surrogate marker, biomarker, fetal surgery, neural tube defects, spina bifida 


\section{Introduction}

Myelomeningocele (MMC) is a clinically important neural tube defect that arises during embryonic development as a result of abnormal primary neurulation. ${ }^{1}$ Common physical problems associated with $\mathrm{MMC}$ include varying degrees of sensorimotor neurological deficit, urogenital and intestinal dysfunction, skeletal malformations and hydrocephalus. The widely accepted concept of a predominantly intrinsic aetiology for the sensorimotor deficit in MMC has recently been challenged by data derived from animal models with surgically created $\mathrm{MMC}^{2-4}$ It was suggested that direct trauma to the exposed fetal spinal cord might occur in utero, thereby eliciting secondary damage to the spinal nerves, so that timely in utero coverage of the MMC may improve the postnatal outcome. ${ }^{2,5,6}$ Although it was suggested previously that a Cesarean section may minimise mechanical stress during labour, and improve postnatal outcome, ${ }^{7}$ severe damage to the exposed spinal cord may have already occurred long before birth. Support for this idea comes from detailed immunohistochemical investigation of surgically created MMC, which demonstrated massive spinal cord astrocytosis at fetal stages, ${ }^{2}$ and electrophysiological examination which suggested substantial axonal loss at similar stages. ${ }^{8,9}$ Both astrocytosis and axonal loss are pathological features which can be monitored by measuring brain-specific proteins. ${ }^{10}$

In the present experimental study we were interested in monitoring the onset and development of the spinal cord injury in MMC at a cellular 
level. The model chosen was the curly tail/loop tail mouse model, which has a high frequency of naturally-occurring spina bifida (SB). ${ }^{11}$ Astrocytosis was estimated by quantifying glial fibrillary acidic protein (GFAP) and S100B. ${ }^{12,13}$ Neuro-axonal injury was estimated by measuring the neurofilament heavy chain $(\mathrm{NfH}) .{ }^{14}$ The study aimed to determine the extent of spinal cord injury in relation to gestational age and size of the SB lesion.

\section{Material and Methods}

Mouse strains and sample collection This study and experimental procedures have been approved by the local ethics committee according to the Declarations of Helsinki and as required under the Animals (Scientific Procedures) Act 1986 of the UK Government. The rules of laboratory animal care (NIH publication No. 86-23) were followed.

Mutant curly tail and loop tail mice were maintained as separate colonies on a $12 \mathrm{~h}$ light-dark cycle (lights on from 07.00 to 19.00). Doubly heterozygous males $(\mathrm{Lp} /+; \mathrm{ct} /+)$ were mated overnight with homozygous curly tail females (+/+; ct/ct) which were checked for copulation plugs the following morning. The day of finding a plug was designated as embryonic day $(\mathrm{E})$ 0.5. Matings generated litters with three different phenotypes: $40.5 \%$ of mice had straight tails, $25.5 \%$ had curly tails, and $34 \%$ had spina bifida (SB), usually with a curly tail. SB animals always showed lesions in the lumbosacral region that extended to the tip of the tail. Experimental litters were collected by sacrificing pregnant females by cervical dislocation at 
E12.5, E14.5, E16.5 and E18.5. The extraembryonic membranes were incised and amniotic fluid was poured directly into a $1.5 \mathrm{~mL}$ Eppendorf tube. Approximately 50 to $100 \mu \mathrm{L}$ of amniotic fluid could be collected per fetus. Samples were immediately snap-frozen in liquid nitrogen. The coded samples were then stored at $-70^{\circ} \mathrm{C}$ until further analysis.

Control animals $(n=134)$ were those that had neither an MMC lesion nor sensorimotor deficit of the hind-limbs. Animals with either a straight or curly tail were included in the control group. MMC lesions were classified as either large or small based on the ratio of total body size to MMC size (see Figure 1 and Table 1). A ratio smaller than 10 indicated a large MMC whereas a ratio larger than 10 represented a small MMC. Of 122 animals with a macroscopically visible SB, the MMC was large in 36 cases and small in 84 cases. Two MMC animals were inadvertently discarded before classification of lesion size.

Brain-specific proteins The phosphorylated neurofilament heavy chain $\left(\mathrm{NfH}^{S M I 35}\right)$, glial fibrillary acidic protein (GFAPSMI26) and S100B were quantified as described. ${ }^{12-14}$ The structure of these proteins is largely conserved across species and the assays have previously been used on tissue from another mouse model. ${ }^{10}$ Due to the small sample volume a maximum of two BSP could be measured per animal.

Statistical analysis All statistical analyses were performed and graphs prepared using SAS software (version 8.2, SAS Institute, Inc., Cary, North 
Carolina, USA). Because of non-Gaussian distribution of the BSP data, the median values and the $25-75 \%$ interquartile range (IQR) are shown. Independent variables were compared using the non-parametric Wilcoxon test. If significance was based on small numbers the results were checked by the Fisher's exact test. The cut-off for categorical data analysis was set to the $100 \%$ cumulative frequency of the indicated control group. The linear correlation between continuous variables was evaluated using the Spearman correlation coefficient $(\alpha=0.05)$. Linear regression analysis was performed using the least-squares method. $\mathrm{P}-$ values $<0.05$ were considered as significant.

\section{Results}

In control mice there was no correlation between gestational age and either GFAP or NfH levels. In contrast S100B concentration increased with age in control mice $(R=0.58, p<0.01)$. This correlation was caused by a significant increase in S100B between E16.5 and E18.5 $(p<0.01)$.

GFAP The concentration of amniotic fluid GFAP differed significantly between control and $S B$ groups $\left(F_{7,153}=37.89, p<0.001\right.$, Figure 2). Post-hoc analysis revealed that this was due to a 100-fold increase of GFAP level in SB mice at E16.5 ( $p<0.001$, Table 2). At E18.5 GFAP levels in the amniotic fluid of SB mice were still approximately 50-fold higher compared to controls $(p<0.001)$. 
The analysis of GFAP concentration in relation to SB lesion size did not reveal any significant difference between large and small MMC lesions.

S100B The concentration of amniotic fluid S100B differed significantly between control and SB groups $\left(F_{7,99}=7.62, p<0.001\right)$. Post-hoc analysis revealed a significant difference between SB and control mice at E18.5 $(p<0.05$, Table 1$)$, but not at earlier stages. There was a correlation $(R=0.69, p<0.01)$ between S100B and GFAP in SB $(n=16)$ but not in control $(n=16)$ mice.

The analysis of S100B concentration in relation to SB lesion size did not reveal any significant difference between large and small MMC lesions.

NfH The concentration of amniotic fluid $\mathrm{NfH}$ differed significantly between control and SB groups $\left(F_{7,91}=3.66, p<0.01\right)$. Post-hoc analysis revealed a significant difference between SB and control mice at E16.5 $(p<0.01)$ and at E18.5 $(p<0.01$, Table 2).

Moreover, the analysis of $\mathrm{NfH}$ concentration in relation to $\mathrm{SB}$ lesion size revealed a significant difference $\left(F_{5,37}=7.98, p<0.001\right)$. A large $M M C$ was associated with higher amniotic fluid $\mathrm{NfH}$ levels than a small $\mathrm{MMC}$ lesion at both E16.5 $(p<0.001)$ and E18.5 $(p<0.01$, Figure 3$)$. 


\section{Discussion}

The findings of this study demonstrate a 100-fold increase in amniotic fluid GFAP at E16.5 in SB mice when compared to controls. We interpret this sudden, dramatic increase in GFAP concentration as indirect evidence that spinal cord injury may accelerate at this time.

GFAP levels started to rise in SB mice at E16.5 compared to the relatively uniform levels observed in SB and control mice at E12.5 and E14.5, and in controls at later stages. GFAP levels remained significantly higher in SB compared to control mice at E18.5, but the difference was less marked $(\approx 50$-fold). This could possibly be due to a protein "wash-out pattern" as observed for S100B in human cerebrospinal fluid. ${ }^{15}$ Alternatively it may be caused by post-translational modifications such as citrullination ${ }^{16}$ or aggregate formation, both of which could potentially interfere with the binding characteristics of the capture antibody in the ELISA. Finally, one needs to consider a "burnt-out" pattern, in which the overall loss of astrocytes on E16.5 is so great that further damage to remaining cells at the site of the MMC results in a less marked elevation of GFAP.

In support of our hypothesis, there is evidence that GFAP concentration is also elevated in human amniotic fluid taken from second-trimester pregnancies with spina bifida and other neural tube defects. ${ }^{17-19}$ Interestingly, Van Regemorter et al. ${ }^{19}$ describe a mean GAFP concentration of 1 $\mathrm{ng} / \mathrm{mL}$ in spina bifida pregnancies samples prior to 24 weeks, but a concentration of $3 \mathrm{ng} / \mathrm{mL}$ in amniotic fluids of spina bifida pregnancies beyond 
24 weeks (Table $2 \mathrm{in}^{19}$ ). This finding parallels our observation of an increased GFAP concentration in late-stage mouse fetuses with SB. On the other hand analysis of a large number of human second trimester pregnancies revealed that staining for cells containing GFAP did not improve the diagnostic sensitivity or specificity of amniotic fluid alpha-fetoprotein and acetylcholinesterase.$^{20}$ It may be that the measurement of GFAP protein itself, rather than staining for GFAP positive cells may be a more sensitive technique, particularly if the release of GFAP into the amniotic fluid is secondary to the death of astrocytes at the site of the MMC lesion.

Amniotic fluid S100B levels have not been investigated systematically with regard to spina bifida, but one group found S100B to be of use in general prenatal screening. ${ }^{21,22}$ In the present study there was a moderate, but significant, increase in amniotic fluid S100B at E18.5, although this was not confined to SB animals. The finding of an increase in S100B concentration at late gestation even in control amniotic fluids suggests a possible physiological role for this protein. Indeed, S100B has cytokine properties which can be neurotrophic at certain concentrations (reviewed $\mathrm{in}^{23}$ ). Additionally, the measurement of $\mathrm{S} 100 \mathrm{~B}$ from body fluids might reflect sources other than the nervous system, such as placenta, adipose tissue, testis and skin. ${ }^{21,24-26}$ The finding of a correlation between S100B and the relatively specific astrocytic marker GFAP $(R=0.69)$, however, suggests that the rise of $\mathrm{S100B}$ in late gestation may be at least in part due to activation of the glial system. The pathological role of the glial system in 
SB has not been investigated in detail and we would like to speculate that preservation of tissue homoeostasis may be one important function. Additionally astrocytic hypertrophy is observed rapidly after axonal injury, ${ }^{27}$ which might be of relevance in SB, as seen below.

The results for $\mathrm{NfH}$ were similar to those for GFAP, with a significant increase in amniotic fluid $\mathrm{NfH}$ being observed in SB fetuses at E16.5 and E18.5. Moreover, the concentration of $\mathrm{NfH}$ correlated directly with the size of the MMC, at both E16.5 and E18.5. Hence, NfH levels were significantly higher in mice with a large SB lesion than in those with a small MMC. In previous human studies, concentrations of $\mathrm{NfH}$ in the cerebrospinal fluid and plasma have been related to disability on clinical scales. ${ }^{28-31}$ Moreover, in a mouse model of chronic experimental autoimmune encephalomyelitis, the $\mathrm{NfH}$ content of tissue homogenate was related to spinal cord atrophy. ${ }^{10}$ In a study focused on neuroprotection, $\mathrm{NfH}$ was used as a secondary outcome measure and correlated with motor function. ${ }^{32}$ The present results suggest that the $\mathrm{NfH}$ concentration in amniotic fluid might provide a tool to estimate the extent of axonal loss in vivo in SB pregnancies.

In utero surgery is now offered as a clinical treatment for meningomyelocele, ${ }^{6}$ although the optimal timing of the intervention remains a critical issue. ${ }^{5}$ This and other aspects of human in utero surgery for MMC are currently being investigated by a multicentered prospective study in the USA: Management of Myelomeningocele Study (MOMS, www.spinabifidamoms.com). 
On the basis of the present results, it would be important to establish cutoff levels related to fetal-age for GFAP and $\mathrm{NfH}$ in normal human amniotic fluid. In pregnancies with fetal SB a slight increase of GFAP and $\mathrm{NfH}$ would be in keeping with the expected slowly progressive pathology, and would indicate that surgery might be a viable option. On the other hand, once an accelerating increase in BSP concentration was observed, suggestive of dramatic tissue loss, it might be considered that in utero coverage would offer only limited benefits, and should be weighed carefully against the risks for mother and child.

\section{Acknowledgements}

The authors thank Professor E.J. Thompson for reading the manuscript. This research was supported by a research fellowship from the University of Zurich (D. Stiefel) and by the Wellcome Trust and the Medical Research Council, UK (A. Copp). 


\section{References}

[1] AJ Copp, ND Greene, and JN Murdoch. The genetic basis of mammalian neurulation. Nat Rev Genet, 4:784-793, 2003.

[2] M Meuli, C Meuli-Simmen, CD Yingling, et al. Creation of myelomeningocele in utero: a model of functional damage from spinal cord exposure in fetal sheep. J Pediatr Surg, 30:1028-1032, 1995.

[3] J Yoshizawa, L Sbragia, BW Paek, et al. Fetal surgery for repair of myelomeningocele allows normal development of anal sphincter muscles in sheep. Pediatr Surg Int, 20:14-8, 2004.

[4] S Bouchard, MG Davey, NE Rintoul, et al. Correction of hindbrain herniation and anatomy of the vermis after in utero repair of myelomeningocele in sheep. J Pediatr Surg, 38:451-408, 2003.

[5] M Meuli, C Meuli-Simmen, et al. The spinal cord lesion in human fetuses with myelomeningocele: Implications for fetal surgery. $\mathrm{J} \mathrm{Pe}$ diatric Surgery, 32:448-452, 1997.

[6] NS Adzick, LN Sutton, TM Crombleholme, and AW Flake. Successful fetal surgery for spina bifida. Lancet, 352:1675-1676, 1998.

[7] DA Luthy, T Wardinsky, DB Shurtleff, KA Hollenbach, DE Hickok, DA Nyberg, and TJ Benedetti. Cesarean section before the onset of labor and subsequent motor function in infants with meningomyelocele diagnosed antenatally. N Engl J Med, 324:662-606, 1991. 
[8] CD Yingling, C Meuli-Simmen, M Meuli, GB Timmel, M Harrison, and NS Adzick. Experimental fetal neurosurgery: effects of in-utero manipulations on somatosensory evoked potentials. Pediatr Surg Int, 15:535-509, 1999.

[9] CD Yingling, C Meuli-Simmen, M Meuli, GB Timmel, NS Adzick, and $M$ Harrison. Assessment of sensory function in neonatal sheep with somatosensory evoked potentials: methodology and normative data. Pediatr Surg Int, 15:530-504, 1999.

[10] A Petzold, D Baker, G Pryce, et al. Quantification of neurodegeneration by measurement of brain-specific proteins. J Neuroimmunol, 138:45-48, 2003.

[11] D Stiefel, T Shibata, M Meuli, PG Duffy, and AJ Copp. Tethering of the spinal cord in mouse fetuses and neonates with spina bifida. $J$ Neurosurg Spine, 99:206-213, 2003.

[12] A Petzold, G Keir, Green AJE, G Giovannoni, and EJ Thompson. An ELISA for glial fibrillary acidic protein. J Immunol Meth, 287:169-177, 2004.

[13] AJE Green, G Keir, and EJ Thompson. A specific and sensitive ELISA for measuring S-100b in cerebrospinal fluid. J Immunol Meth, 205:3541, 1997. 
[14] A Petzold, G Keir, Green, et al. A specific ELISA for measuring neurofilament heavy chain phosphoforms. J Immunol Methods, 278:179190, 2003.

[15] A Petzold, G Keir, D Lim, M Smith, and EJ Thompson. CSF and serum S100B: release and wash-out pattern. Brain Res Bull, 61:281285, 2003.

[16] AP Nicholas, T Sambandam, JD Echols, and WW Tourtellotte. Increased citrullinated glial fibrillary acidic protein in secondary progressive multiple sclerosis. J Comp Neurol, 473:128-36, 2004.

[17] M Albrechtsen, A Massaro, and E Bock. Enzyme-linked immunosorbent assay for the human glial fibrillary acidic protein using a mouse monoclonal antibody. J Neurochem, 44:560-506, 1985.

[18] M Albrechtsen, E Bock, and B Norgaardpedersen. Glial fibrillary acidic protein in amniotic fluids from pregnancies with fetal neuraltube defects. Prenatal Diagnosis, 4:405-410, 1984.

[19] N Van Regemorter, J Gheuens, M Noppe, et al. Value of glial fibrillary acidic protein determination in amniotic fluid for prenatal diagnosis of neural tube defects. Clin Chim Acta, 165:83-8, 1987.

[20] JE Bell, L Barron, and G Raab. Antenatal detection of neural tube defects: comparison of biochemical and immunofluorescence methods. Prenat Diagn, 14:615-622, 1994. 
[21] P Florio, F Michetti, M Bruschettini, et al. Amniotic fluid S100B protein in mid-gestation and intrauterine fetal death. Lancet, 364:270-202, 2004.

[22] F Michetti and D Gazzolo. S100B testing in pregnancy. Clin Chim Acta, 335:1-7, 2003.

[23] R Donato. S100: a multigenic family of calcium-modulated proteins of the EF-hand type with intracellular and extracellular functional roles. Int J Biochem Cell Biol, 33:637-668, 2001.

[24] F Michetti, L Lauriola, M Rende, VM Stolfi, F Battaglia, and D Cocchia. S-100 protein in the testis. An immunochemical and immunohistochemical study. Cell Tissue Res, 240:137-42, 1985.

[25] K Takahashi, T Isobe, Y Ohtsuki, T Akagi, H Sonobe, and T Okuyama. Immunohistochemical study on the distribution of alpha and beta subunits of S-100 protein in human neoplasm and normal tissues. Virchows Arch B Cell Pathol Incl Mol Pathol, 45:385-396, 1984.

[26] H Hidaka, T Endo, S Kawamoto, E Yamada, H Umekawa, K Tanabe, and $\mathrm{K}$ Hara. Purification and characterization of adipose tissue S100b protein. J Biol Chem, 258:2705-2709, 1983.

[27] I Reisert, G Wildemann, D Grab, and C Pilgrim. The glial reaction in the course of axon regeneration: a stereological study of the rat hypoglossal nucleus. J Comp Neurol, 10:121-128, 1984. 
[28] JN Lycke, JE Karlsson, O Andersen, and LE Rosengren. Neurofilament protein in cerebrospinal fluid: a potential marker of activity in multiple sclerosis. J Neurol Neurosurg Psychiatry, 64:402-404, 1998.

[29] YK Semra, OA Seidi, and MK Sharief. Heightened intrathecal release of axonal cytoskeletal proteins in multiple sclerosis is associated with progressive disease and clinical disability. J Neuroimmunol, 122:132109, 2002.

[30] A Petzold, MJ Eikelenboom, G Keir, et al. Axonal damage accumulates in the progressive phase of multiple sclerosis: A 3-year followup study. J Neurol Neurosurg Psychiatry, 76:206-211, 2005.

[31] A Petzold, K Rejdak, and GT Plant. Axonal degeneration and inflammation in acute optic neuritis. J Neurol Neurosurg Psychiatry, 75:1178-1780, 2004.

[32] G Pryce, Z Ahmed, DJ Hankey, et al. Cannabinoids inhibit neurodegeneration in models of multiple sclerosis. Brain, 127:2191-2202, 2003. 
Table 1: Classifying MMC size in curly tail/loop-tail fetuses. Lengths shown are the ranges typically seen in the study. Ratio = Total body length $/ \mathrm{MMC}$ length .

\begin{tabular}{|c|c|c|c|}
\hline \multirow[b]{2}{*}{ Size } & \multicolumn{2}{|c|}{ Gestational age } & \multirow[b]{2}{*}{ Ratio } \\
\hline & E16.5 & E18.5 & \\
\hline Total body length (mm) & $15-18$ & $26-28$ & - \\
\hline Large MMC length $(\mathrm{mm})$ & $3-5$ & $4-6$ & $<10$ \\
\hline Small MMC length $(\mathrm{mm})$ & 1.5 & 2 & $>10$ \\
\hline
\end{tabular}




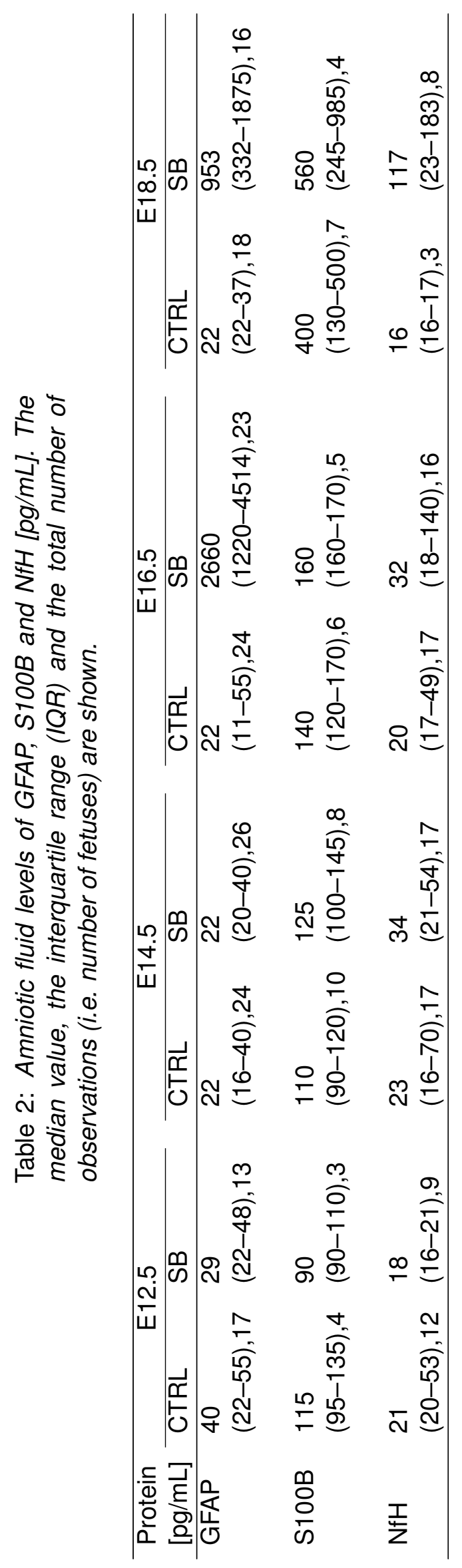


(A)

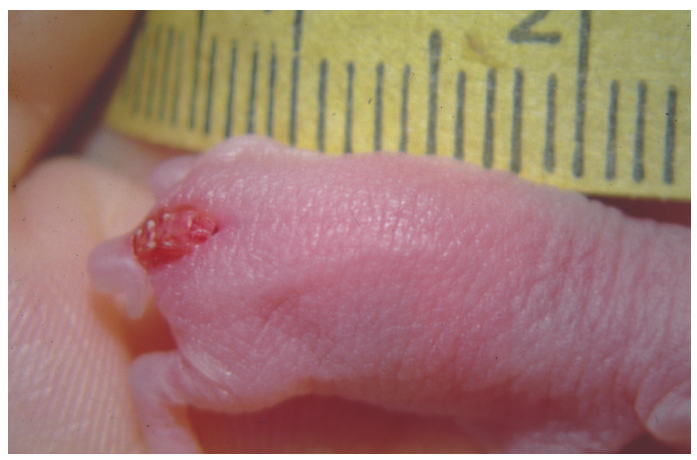

(B)

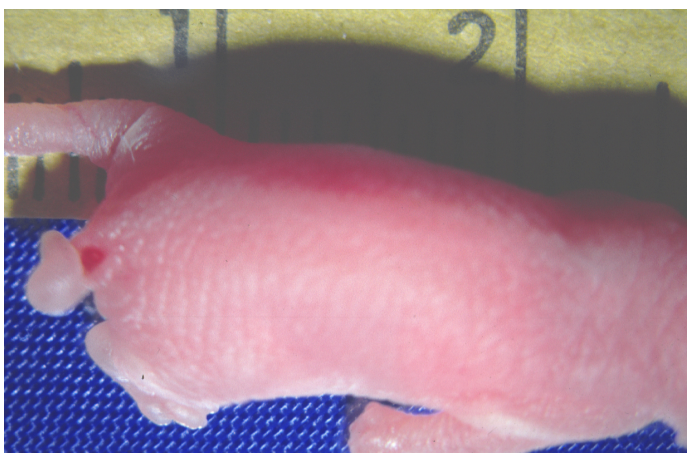

Figure 1: The curly tail/loop tail mouse model. Large (A) and small (B) $M M C$ lesions are shown. See Table 1 for a precise definition of MMC size. 


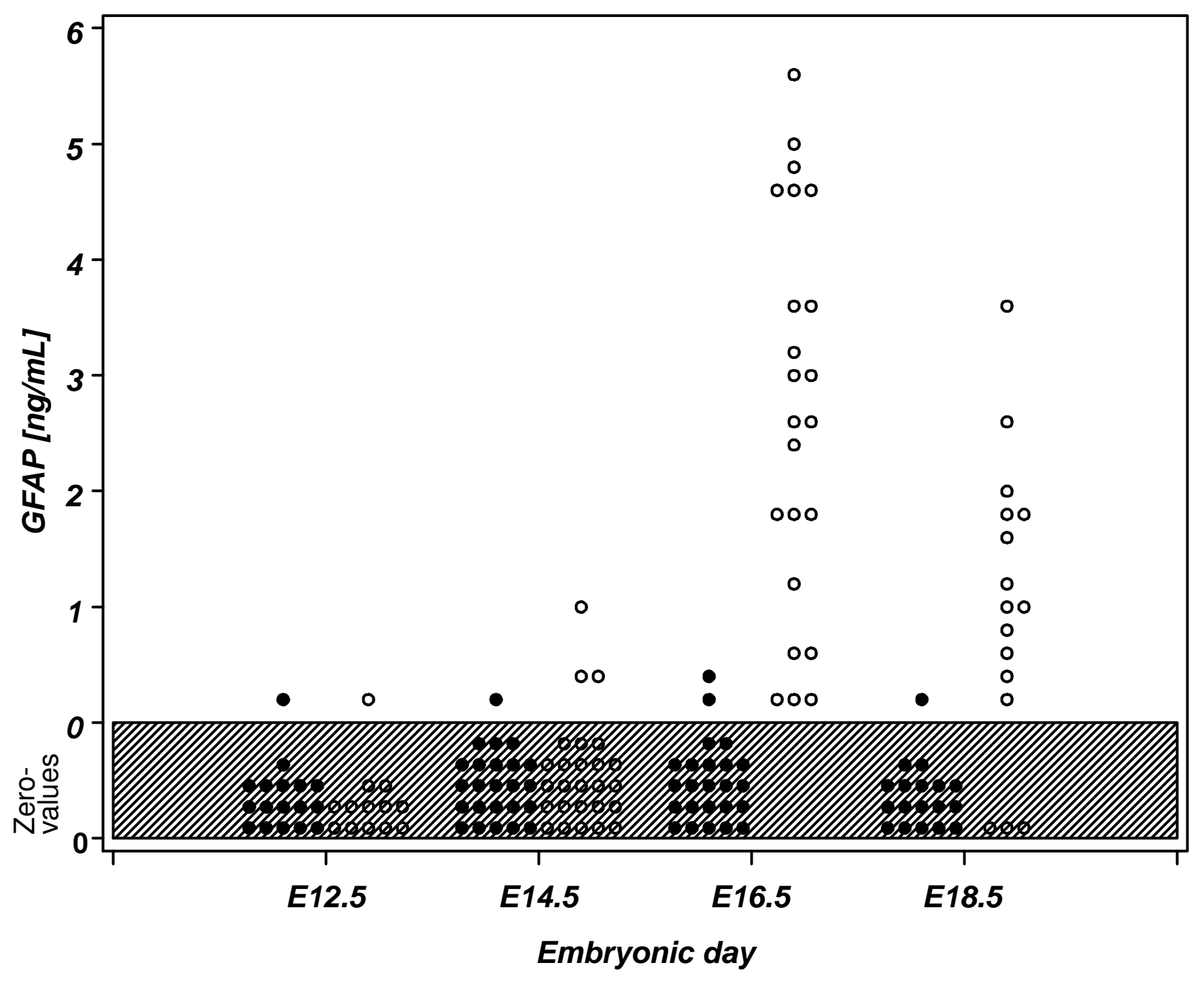

- - CTRL 0 ○ $0 \quad S B$

Figure 2: Amniotic fluid levels of GFAP in control (closed circles) and SB mice (open circle). Note that for clarity of the figure, all zero values are plotted in the hatched area and concentrations are presented in [ng/mL], the text and Table 2 however, refer to $[\mathrm{pg} / \mathrm{mL}]$. 


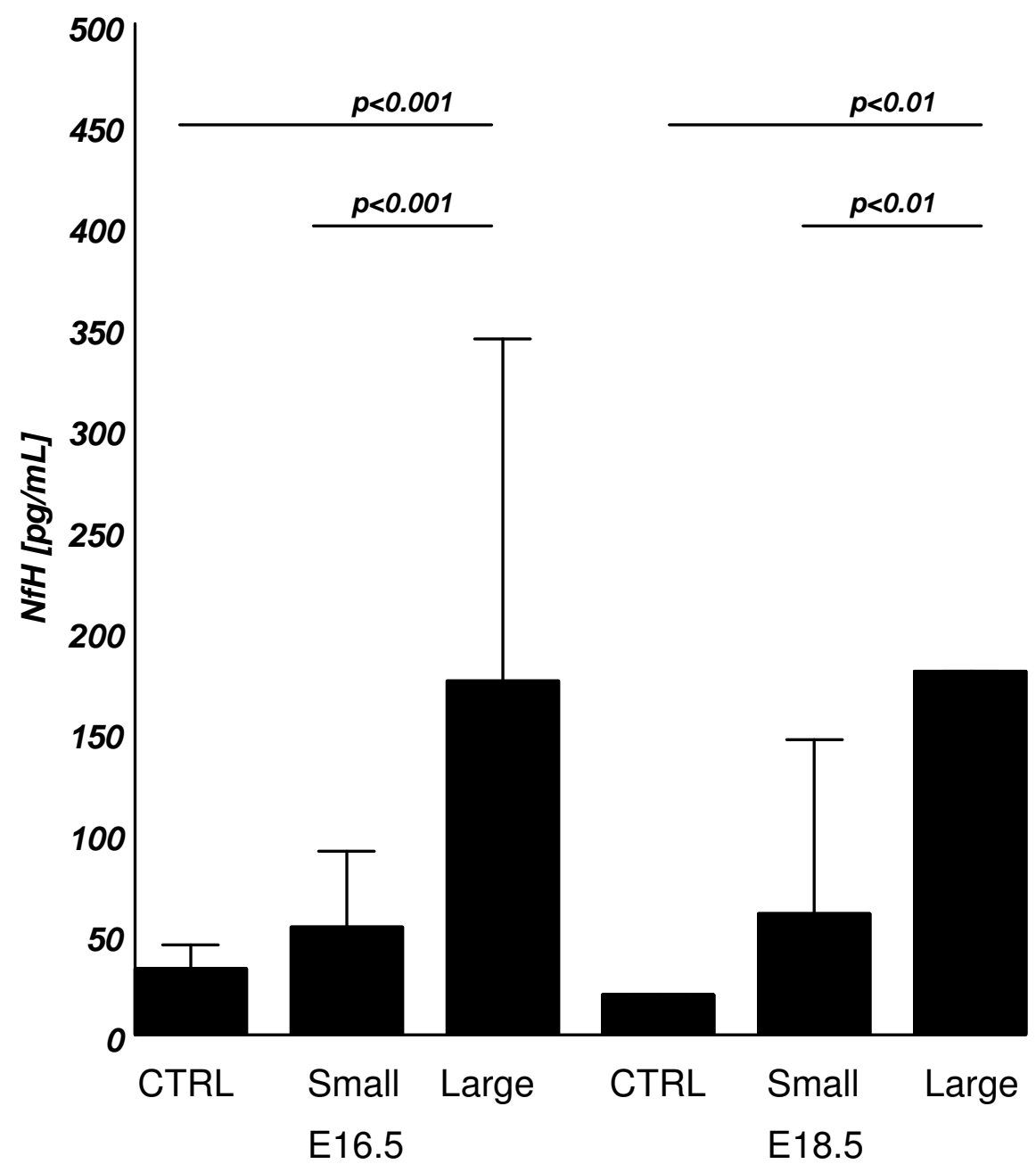

Figure 3: Mean ( \pm 1 standard deviation) amniotic fluid levels of $\mathrm{NfH}$ in control (CTRL), SB mice with small MMC and SB mice with large MMC at E16.5 and E18.5. There was no statistically significant difference between the NfH concentration in small MMC and controls. 